\title{
Chronic rhinosinusitis in children: therapeutical update
}

\begin{abstract}
Introduction: Chronic rhinosinusitis (CRS) is an inflammatory disease that affects the mucosa of the nose and the sinuses, and lasts at least 12 weeks. This condition is common in children and the treatment prevents recurrences and complications. The treatment is initially clinical. In case of therapeutic failure we can resort to surgical treatment
\end{abstract}

Objectives: To evaluate the main therapeutic methods, both clinically and surgically. Among the available surgeries, we will approach: adenoidectomy, Balloon Catheter Sinuplasty and Functional Endoscopic Sinus Surgery.

Methods: A literature review was conducted, with research in databases: PUBMED and SCIELO. Seventeen articles were found and analyzed. Results: The antibiotic therapy lasts, on average, 4 weeks. Adenoidectomy is the first choice for surgical treatment of chronic rhinosinusitis, especially in younger children, but exhibited effectiveness only in half of the patients. Functional Endoscopic Sinus Surgery is the best option in children older than 6 years and did not show significant change in facial growth. Balloon Catheter Sinuplasty assists and complements Functional Endoscopic Sinus Surgery, but could not replace it. For being less invasive, it is very effective and has fewer complications.

Conclusion: Chronic rhinosinusitis is a common problem in children, and is often associated with: asthma, allergic rhinitis, nasal polyposis, among others. When clinical treatment failure occurs, surgical treatment should be considered, but it should be in accordance with the indications for each type of pathological condition, and be as invasive as possible. Among the surgical treatments available, including the adenoidectomy, Functional Endoscopic Sinus Surgery and Balloon Catheter Sinuplasty are effective and safe procedures, with few complications.

Keywords: chronic rhinosinusitis, treatment, functional endoscopic sinus surgery, FESS, quality of life, adenoidectomy, balloon catheter sinuplasty, BCS, sinus, children, nasal obstruction
Volume 10 Issue 4 - 2018

\author{
Carlos Eduardo Borges Rezende, Isabel \\ Marrach de Pasqual, Lais Tamie Hiroshima, \\ Tifani Dawidowicz Fernandes, Stephanie \\ Rissio \\ Otolaryngologist, Brazil
}

Correspondence: Valentine Pankowski, Department of Chalcolithic and the Bronze Age Archaeology, The National Academy of Sciences of Ukraine, Ukraine, Tel (044) 4I8-9195, Fax (044) 4l8-3306, Email vpns@ukr.net

Received: June 20, 2017 | Published: July 19, 2018

\section{Introduction}

Rhinosinusitis is an inflammatory and infectious condition that affects the paranasal sinuses and nasal mucosa, preceded in many cases by rhinitis symptoms, such as rhinorrhea, sneezing, obstruction and nasal pruritus. ${ }^{1}$

The pediatric population has an average of 6 to 8 episodes of colds a year, and 0.5 to $5 \%$ of these develop into bacterial rhinosinusitis. ${ }^{2}$ It can be classified according to the duration of symptoms in acute, up to four weeks; Subacute, from four to 12 weeks, and chronic, greater than 12 weeks. There is also recurrent acute rhinosinusitis in which each episode lasts less than 30 days, with complete resolution between the episodes and a period of at least 10 days between them. ${ }^{1}$

The main causes of chronic rhinosinusitis are: infectious viral, fungal and bacterial (Streptococcus pneumoniae, Haemophilus influenzae, Staphylococcus aureus, Streptococcus beta and alpha hemolytic, Bacteroides spp., Fusobacterium and gram positive cocci), nasal polyps, cystic fibrosis, allergy, immunodeficiency, Primary and secondary ciliary dyskinesia, gastroesophageal and laryngopharyngeal reflux, asthma, adenoid hypertrophy, nasal septum deviation and mechanical obstruction of the meatal ostium. ${ }^{1}$
The mechanism that has been proposed for the pathophysiology of rhinosinusitis is due to the narrow ostium caliper, which predisposes to obstruction. This obstruction may be mechanical or inflammatory. Mechanics occur due to choanal atresia, adenoid hypertrophy, nasal septum deviation, nasal polyps, foreign body and tumors. On the other hand, inflammation includes: IVAS, immunodeficiencies, PDD and drug rhinitis. These mechanisms obstruct the drainage of sinus ostia, modifying ventilation and ciliary clearance, leading to stasis of mucus in the paranasal sinuses, leading to bacterial growth, leading to mucosal inflammation. ${ }^{1}$

Chronic rhinosinusitis results mainly from inappropriately treated or untreated acute rhinosinusitis. The typical clinical picture of this condition is characterized by nasal obstruction, antero-posterior mucopurulent rhinorrhea, post nasal drip, cough and hyposmia. There may be facial pain, which, however, is uncommon. ${ }^{2}$

The diagnosis is based on the clinical history. The main clinical findings are edema and nasal mucosa enigma and "candle signal" (presence of mucopurulent retropharyngeal secretion), demonstrating post nasal drip. ${ }^{3}$

Among the confirmatory methods regarding the diagnosis of CRS, nasofibroscopy and imaging can be used. Nasofibroscopy 
allows visualization of secretion from the meatus, as well as mucosal edema. CT imaging is the gold standard, allowing visualization of the obstruction of the ostium-meatal complex, as well as concealment or thickening of the paranasal sinus mucosa, allowing a mapping of the anatomy, besides being important in the surgical planning of the patient. MRI is considered the best technology available to evaluate the anatomy, but it is useful only when multiple or extensive sinus complications are suspected. Radiography of the paranasal sinuses has been widely used, however the advantages of CT and MRI have made this examination lose space, as it occurs in overlapping structures, as well as difficulty in accessing the anterior ethmoid sinus, the middle meatus And infundibular and air passages of the frontal recess. ${ }^{3}$

When acute rhinosinusitis is treated properly, it evolves to cure in most cases; however, it can become chronic, especially if not treated properly. The drug treatment of CRS is performed by means of antibiotic therapy. In the case of therapeutic failure, one may consider the surgical approach, which is based on cure of the cause (eg adenoidectomy), BCS (Balloon Catheter Sinuplasty) or FESS (Functional Endoscopy Sinus Surgery). ${ }^{2}$ FESS is a minimally invasive technique that approaches the paranasal sinuses in order to provide maximum preservation of the nasosinusal mucosa as well as restoring ventilation and drainage. ${ }^{4} \mathrm{BCS}$ uses the principle of dilatation of sinus ostia by catheter-guided flasks. It is an alternative to the minimally invasive treatment of patients with CRS refractory to clinical treatment.

\section{Objectives}

The general objective of this literature review is to study the possible treatments for chronic rhinosinusitis, aiming to establish the best therapy, with fewer side effects and fewer complications.

\section{Methods}

A literature review was carried out, with research in the databases PUBMED and SCIELO. We found 17 articles that met the criteria for inclusion: articles of the last 25 years on the treatment of chronic rhinosinusitis in children up to 14 years of age. The articles searched were limited to Portuguese and English only. None of the 17 articles found were excluded from this review. The search was performed using the following keywords: chronic rhinosinusitis, treatment, functional endoscopic sinus surgery (FESS), quality of life, adenoidectomy, Balloon Catheter Sinuplasty BCS), paranasal sinuses (sinus), children and nasal obstruction. The terms listed above were used together or in isolation. Of the 17 articles found, we sought to address in our study systematic reviews, clinical trials and Meta-analyzes.

\section{Literature review}

Vlastarakos et al., ${ }^{5}$ carried out in their study a systematic analysis and meta-analysis of published intervention studies, in which they evaluated the effectiveness of FESS as treatment of CRS in children and their impact on their quality of life.

Thirty-one studies were found and analyzed, and after exclusion of those who did not meet the criteria, four prospective studies, five comparative retrospectives and six retrospective studies were systematically analyzed. In all, 1301 patients participated in the study. The 13 groups that evaluated the effectiveness of FESS, concluded that this is an effective treatment for CRS in children. In addition, five studies concluded that FESS is associated with improvement in the quality of life of this patients. ${ }^{5}$ The use of FESS in the pediatric population seems to be less effective in the presence of associated systemic disease, requiring operative revisions. ${ }^{5}$

Most of the treated patients were followed for more than one year. The incidence of major complications was $0.6 \%$ and the others were considered minor complications. Table 1 refers to FESS complications in the studied pediatric population. ${ }^{5}$

Table I Complications of FESS in the pediatric population-20I3

\begin{tabular}{ll}
\hline Severity & Type \\
\hline \multirow{3}{*}{ Bigger } & Bleeding $(n=5)$ \\
& CSF leakage $(n=1)$ \\
& Meningitis $(n=2)$ \\
& Papyraceous lamina rupture $(n=2)$ \\
Less & Bleeding $(n=7)$ \\
& Meatal stenosis $(n=13)$ \\
\hline
\end{tabular}

Source: (Clinical Pediatrics, 2013).

Fiocchi et al., ${ }^{6}$ have done a study on the topical treatment of rhinosinusitis, since this is a very used modality in children. In all, 52 studies were used, which focused on or included children. Nasal irrigation, nasal decongestants, corticoids, antibiotics, antifungals and antihistamines are used on a large scale and were analyzed in this study.

Nasal irrigation: used with nasal solution, is prescribed routinely. Several mechanisms of action were proposed, but there was no consensus on its use in rhinosinusitis in the pediatric population. Its prophylactic use in RSC exacerbations is empirical and is not supported by controlled clinical data. ${ }^{6}$ Topical decongestants: three studies have suggested that, in children old enough to cooperate, topical decongestants may be helpful in rhinosinusitis; this medication decreases mucus viscosity and nasal bleeding. Prospective studies are lacking and are required to assess the efficacy of $\alpha$-adrenergic agents in the prevention or treatment of this condition. ${ }^{6}$

Topical antihistamines: there are no data recommending the use of topical antihistamines in acute bacterial rhinosinusitis, however, these drugs may play a significant role in CRS, especially in patients with allergic rhinitis. Topical antibiotics: in six randomized studies, comparisons were made between antibiotics for rhinosinusitis in children and standard or placebo treatment, and a small benefit was noted in the use of topical antibiotics. ${ }^{6}$ Antifungals: no studies have been found related to its use, although fungal infection is frequently present in sinus infections. ${ }^{6}$

Cazzavillan et al., ${ }^{7}$ carried out a review article, in which they dealt with the behavior in CRS, due to the fact that their treatment is difficult in children, being frequent recurrences and failures; And there is no consensus, since its exact etiology is still under discussion. After this, it was observed that: when the cause of rhinosinusitis is bacterial, the recommended antibiotic treatment is with high doses of amoxicillin or amoxicillin with clavulanate. In patients allergic to penicillin, clarithromycin or azithromycin may be used. The topical treatment with topical corticosteroids, respecting the age range for its use, and saline solution, hypertonic or not, can also be used.

Nasal endoscopy provides a good assessment of nasal conditions. CT should be associated in all cases of treatment failure, in the suspicion of orbital or intracranial complications and in the planning 
of any surgical procedure. ${ }^{7}$ Adenoidectomy and minimally invasive endoscopic surgical procedures are reserved for cases that could not be clinically resolved, with adenoidectomy being the most commonly performed surgical procedure in children, but its effectiveness is controversial, since randomized trials have failed to prove that, alone, Is able to cure RSC. ${ }^{7}$ Sinusoplasty with balloon had its effectiveness confirmed in $91 \%$ of the cases and there were no complications nor side effects. Since it does not remove tissue or bone, it appears to be a suitable procedure for children.?

FESS was effective in more than $80 \%$ of the cases. Although there is no consensus about when to perform FESS for CRS, this technique is recommended in the following cases: I) failure of maximal drug therapy, adenoidectomy and systemic antibiotic therapy after culture, with persistence of sinonasal disease; II) when anatomical abnormalities predispose to the recurrence of rhinosinusitis, with worsening of quality of life; III) in nasal polyposis, to facilitate the application of topical corticosteroids or as adjuvant in desensitization of aspirin-sensitive patients with orbital or intracranial complications of sinus disease, with the exception of periorbital cellulitis.

Ramadan and Terrell ${ }^{8}$ conducted a prospective, non-randomized study in which 49 children aged 2 to 11 years were divided into two groups, those who underwent BCS and those who underwent adenoidectomy alone. The aim of the study was to find out if the addition of BCS at the time of adenoidectomy improved the outcome. All the children fulfilled the inclusion criteria, age from 2 to 12 years and planned surgical intervention, and were followed for 12 months.

Of the 49 children, 30 underwent BCS and 19 underwent adenoidectomy. The two groups were similar, except for age. Statistically the adenoidectomy group was younger than the BCS group. ${ }^{8}$

Twenty-four children (80\%) who underwent BCS achieved improvement in symptoms, compared to $10(52.6 \%)$ who underwent adenoidectomy. Two children in the BCS group had failure of the surgical intervention and required FESS. Three children in the adenoidectomy group had failed this treatment and performed BCS afterwards. ${ }^{8}$

This study demonstrated that BCS is relatively safe and obtained a better result than those who only underwent adenoidectomy. ${ }^{8}$ Sommer et al., ${ }^{9}$ carried out a study with 44 patients diagnosed with PDD through a questionnaire about diagnosis, clinical history, manifestations and treatment related to otolaryngological symptoms.

The 44 patients or their caregivers answered the questionnaire. The first symptom of PDD appeared in the lungs in $51 \%$, in the nose and in the paranasal sinuses in $35 \%$ and in the ears in $14 \%$ of these patients. ${ }^{9}$ Of the patients who answered the questionnaire, 59\% reported recurrent symptomatology in the paranasal sinuses. Of these, $25 \%$ did not receive antibiotic therapy, $19 \%$ were treated with antibiotics up to 10 times, $24 \%$ up to 30 times and $32 \%$ more than 30 times. ${ }^{9}$ Surgical intervention had to be performed at least once in $69 \%$ of patients with PDS associated with chronic rhinosinusitis, and this procedure should always be indicated very carefully. ${ }^{9}$

Cornet et al., ${ }^{10}$ performed a prospective and retrospective study simultaneously, using a QoL questionnaire applied to children with CRS associated with nasal polyps (PN) who underwent FESS. This study aimed to evaluate the long-term results of FESS in 44 children with CRS associated with PN. Of these, 36 were followed up after FESS and answered the questionnaire.
After FESS, $86 \%$ of the patients had a favorable result and $14 \%$ needed some surgical revision. Based on this, the authors came to the consensus that surgical intervention should be considered in pediatric patients with CRF associated with NP when there is therapeutic failure. This study demonstrated that FESS is a safe and effective treatment in the pediatric population with CS associated with PN. ${ }^{10}$ Sharkawy, Elmorsy and Eladl ${ }^{11}$ conducted a prospective study to analyze the post-operative outcomes of FESS surgery in children and the factors that influence this outcome. A sample of 87 children aged 14 years or younger with different FESS applications was used for the period from 2005 to 2010 . Of these 87 children, 45 presented CRS with allergic rhinitis, 36 had only CRS, and 6 had CRS and associated polyps. In addition to being classified according to the different diseases, they were also classified into groups according to age: group A (up to 5 years), group B (between 6 and 10 years) and group C (between 11 and 14 years). Table 2 refers to the age distribution according to the diseases. ${ }^{11}$

Table 2 Percentage of CRS associated with other diseases in each age group (mean age 9.9 years) -2011

\begin{tabular}{lccc}
\hline Disease & Group A & Group C & Group B \\
RSC with rhinitis (45) & $6(13.3 \%)$ & $10(22.2 \%)$ & $29(64.5 \%)$ \\
RSC (36) & $4(8.9 \%)$ & $20(44.4 \%)$ & $12(26.7 \%)$ \\
RSC with polyp (6) & & & $6(100 \%)$ \\
Total (87) & $10(11.5 \%)$ & $30(34.5 \%)$ & $47(54 \%)$ \\
\hline
\end{tabular}

Source: (European Archives Otorhinolaryngology, 2011).

The patients were submitted to a questionnaire evaluating the degree of symptoms (in absent, mild, moderate and severe), physical examination and nasofibroscopy. The diagnosis of allergic rhinitis was confirmed by anamnesis, clinical examination, positive skin test for a certain allergen and high total serum IgE, and all patients underwent paranasal sinus CT scanning. ${ }^{11}$ The overall success rate of FESS in children in this study was $87.69 \%$, being divided in $87.5 \%$ for children with CRS and allergic rhinitis; $85.7 \%$ in children with only CSR and $93 \%$ in CSR and polyps. Therefore, FESS was shown to be quite effective for the treatment of CRS. ${ }^{11}$

Aanaes et al., ${ }^{12}$ conducted a prospective observational, nonrandomized study of the effect of FESS on the decrease of pathogenic bacteria in cystic fibrosis (CF) patients, following 58 patients for 12 months. Of the 58 patients included in the study, 12 were pulmonary transplants (LTX), 13 with chronic lung infection and 33 patients with intermittent colonization of bacteria in the lung. Considering the 58 patients, there are 116 paranasal sinuses. Table 3 indicates the number of paranasal sinuses that remained infected, in relation to their pairs, after FESS surgery. ${ }^{12}$

Table 3 Number of infected paranasal sinuses after 12 months of FESS surgery in each initial condition

\begin{tabular}{lll}
\hline Pulmonary status & Preoperative & $\begin{array}{l}\text { I2 months after } \\
\text { surgery }\end{array}$ \\
\hline LTX & $\begin{array}{l}24 \text { de } 24 \\
(100 \%)\end{array}$ & 9 de $20(45 \%)$ \\
Chronically infected & 25 of $26(96 \%)$ & 8 of $18(44 \%)$ \\
Colonized intermittently & 55 of $66(83 \%)$ & 12 of $48(25 \%)$ \\
\hline
\end{tabular}

Source: (Modified table of American Journal of Rhinology \& Allergy, 2013). 
In the postoperative period, for all patients, a broad-spectrum antibiotic against the expected bacteria, 6 months of nasal topical steroid, 6 months of nasal lavage with saline and antibiotic (Colistimethate sodium) was administered for 2 weeks. Nose, ears and oropharynx of patients at 1 week, 1, 3 and 12 months prospectively. ${ }^{12}$

This study demonstrated that the FESS associated to clinical postoperative treatment can eliminate the population of bacteria present in the paranasal sinuses in $\mathrm{CF}$ patients, especially those with intermittent lung colonization. ${ }^{12}$

Lai et al., ${ }^{1}$ developed a literature review on the association between CRS and asthma. An algorithm was created for children with uncontrolled asthma, so that the presence of CRS was detected more easily, and the treatment of CRS would improve asthma. Algorithm 1 shows a possible approach for children with difficult asthma control.

This algorithm was shown to be of great importance for the diagnosis of CRS in asthmatic children and to treat them correctly, aiming to improve asthmatic symptoms. ${ }^{1}$

\section{Discussion}

"Regarding the clinical treatment of CRS, the use of antibiotics for a period of three to four weeks seems to present a more satisfactory cure rate than when used for regular time (10 to 14 days)". ${ }^{13}$

However, for Vlastarakos et al., "the treatment of choice for CRS should include two to six weeks of antibiotic therapy in addition to treatment of any concomitant disease." In the study by Martinez \& Simon ${ }^{14}$, chronic macrolide therapy showed an anti-inflammatory effect, leading to improved ventilation in the maxillary, ethmoidal, sphenoid and frontal sinuses, as well as improving symptoms such as rhinorrhea and post nasal drip in $44 \%$ Of patients. This therapy has also been shown to decrease neutrophil recruitment and the level of IL-8 in nasal secretion.

Pignatary et al., ${ }^{13}$ stated that the use of antihistamines, corticosteroids, decongestants and fluidizing agents was not proven effective. Topical or systemic decongestants have utility reserved for the first few days of treatment to relieve obstructive symptoms. Antihistamines and corticosteroids are generally used in patients who have a baseline allergy and are given orally for a short time.

The child presenting CRS resistant to adequate clinical treatment and the treatment of concomitant diseases has a relative surgical indication

Nasosinusal surgery has developed rapidly in the last two decades, leveraged mainly by the introduction of endoscopes into its practice. It was originally conceived as a functional surgical treatment of chronic rhinosinusitis (associated or not with nasosinusal polyposis) refractory to clinical treatment. ${ }^{4}$ Surgical treatment in children should be performed with caution and be the least invasive. ${ }^{13}$

Table 4 presents the most common indications for endoscopic nasosinusal surgery. Among the surgical procedures, FESS, BCS and adenoidectomy stand out.

Adenoidectomy has been advocated as the first surgical treatment option in children with CRS because of the excellent safety and ease of technique. This surgery serves to eradicate the reservoir of bacteria and improve the permeability of the nasal fossae, increasing ventilation and drainage. ${ }^{2}$ According to Ramadan \& Terrel $^{8}$, the adenoidectomy proposed as the main surgery for the treatment of CRS is effective in only half of the children. This surgery has been shown to be less effective in children with asthma or less than 6 years of age.

Table 4 Most Common Indications for Endoscopic Nasosinusal

Surgery-2006

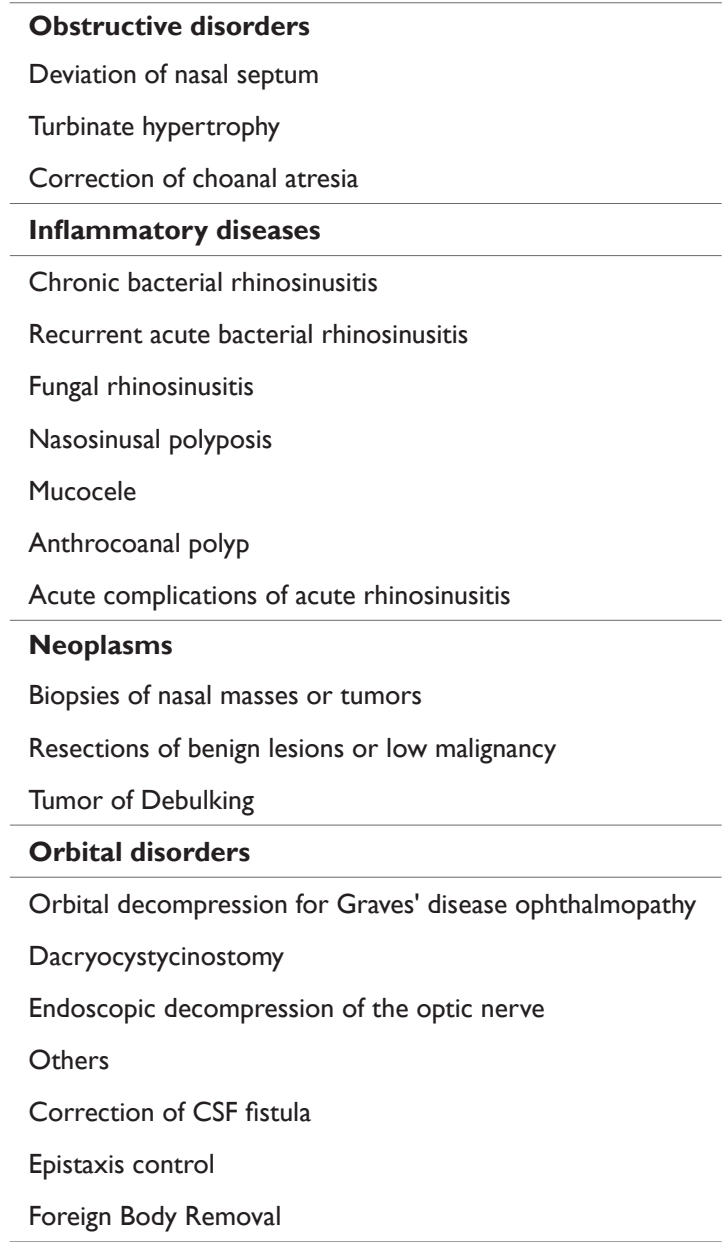

Source: (Brazilian Journal of Video Surgery, 2006).

Children with asthma or younger than 7 years of age adenoidectomized, required revision surgery earlier. ${ }^{8}$ Balloon sinusoplasty is an alternative in the treatment of chronic rhinosinusitis. It is not a substitute for functional surgery of the paranasal sinuses. The use of the combined balloon to the endoscopic surgical approach may minimize surgical complications such as cicatricial stenosis, especially in regions such as the recess of the frontal. ${ }^{8}$

BCS appears to be a viable, safe and efficient technology in selected patients. It is presented as an auxiliary and complementary therapy to the FESS, mainly in the disease related to the frontal recess. It also has a good perspective in febrile ICU patients with sinus focus and patients with high anesthetic and bleeding risk. ${ }^{8}$

Brown et al. (2006), after performing balloon dilation in 10 patients, in a total of 18 breasts, considered that the easiest breast to cannulate was the sphenoid, followed by the frontal, the maxilla being the most Difficult because of the large angulation of the guide catheter necessary to transpose the posterior end of the uncinate process towards the ethmoidal infundibulum and its natural ostium. This study did not report any major complications such as cerebrospinal fluid 
leakage. It demonstrated a high degree of mucosal preservation and a low degree of bleeding, concluding that this method is relatively safe and feasible.

Brown et al. (2006) suggest that caution should be exercised in patients with signs of bone neoformation, since an attempt to dilate may not be effective and there is a risk that, if exaggerated pressure is used, it will be transmitted To adjacent structures such as the base of the skull and the orbit. Care should also be taken during the procedure to avoid postoperative complications such as lateralization of the middle concha and synechiae. To avoid this complication the authors suggest the use of absorbable gel in the middle meatus after the procedure.

The FESS approaches the paranasal cavities providing maximum preservation of the nasosinusal mucosa as well as restoring ventilation and drainage. This procedure optimizes wound healing, prevents unnecessary removal of growing bones and has simple postoperative care, besides being well accepted, widespread and presenting excellent results. ${ }^{4}$ However, cicatricial stenoses secondary to surgical manipulation still occur, even in the hands of experienced surgeons, and especially in narrow regions such as the frontal recess. ${ }^{5}$

Considering the concern about the impact of surgery on facial development, Bothwell et al. (2002), VLASTARAKOS et al. (2013) performed a quantitative and qualitative anthropomorphic analysis using 12 patterns of facial measures, which did not show a statistically significant change in facial development between the children who performed FESS and those who did not.

Sharkawy, Elmorsy and Shawky ${ }^{11}$ verified that the improvement in the postoperative period was related to: preoperative disease extension, adenoidectomy and a second surgical approach. The absolute indications for the FESS in the pediatric population are: I) complete nasal obstruction in cystic fibrosis due to massive polyposis or due to the lateral wall of the medialized nose; II) orbital abscess; III) intracranial complications; IV) antro-coanal polyp; V) mucocele and VI) fungal rhinosinusitis. ${ }^{5}$

It was observed that the FESS result changes according to the age of the group, and in younger children there was a greater adhesion and more recurrences, and in the older ones it was related to a greater loss of blood and a significant surgical improvement. It was also found that patients with asthma showed an insignificant improvement with FESS. ${ }^{11}$ According to Lai et al., ${ }^{1}$ CSR and asthma are generally found in association, and may be the continuous manifestation of upper and lower airway inflammation. This has led to the concept of airways disease. The idea behind this is that both share similarities in histology, physiology, clinical and immune response, as well as allergic and nonallergic factors. Several experiments support this concept. Thus, it is concluded that the treatment of CRS influences an improvement in asthma.

The main surgical options for CSR in improving asthma are adenoidectomy, FESS, and adenoidectomy with FESS. The association of the latter two showed a better result, since adenoidectomy is important for the removal of adenoid tissue, which functions as a bacterial reserve, interfering with mucociliary clearance. Because FESS offers a risk of facial growth retardation, physicians prefer to perform adenoidectomy earlier, considered safer. ${ }^{1}$

For Ramadan, ${ }^{2}$ FESS should be the treatment of choice for older children, who did not obtain results with clinical treatment and for those whose rhinosinusitis presented complications. For younger children, adenoidectomy should be given priority.

Aanaes et al., ${ }^{15}$ in their study showed that FESS associated with a postoperative clinical treatment can eradicate the bacteria present in the paranasal sinuses in CF patients. Therefore, the frequency of bacterial colonization in the lung is reduced with FESS, especially in patients with intermittent lung colonization. In a study conducted by, ${ }^{4}$ regarding patient satisfaction with interventions: $91.4 \%$ answered "yes, they would redo the procedure" for sinusoplasty, against $48.6 \%$ of patients submitted to FESS. The number of days that patients used analgesic medication was also significantly lower in patients undergoing sinusoplasty $(0.80 \pm 0.72$ days $)$ versus FESS patients $(1.34 \pm 0.99$ days; $\mathrm{p}=0.011)$.

The costs of sinusoplasty were approximately US $\$ 12,656.57 \pm$ $3,184.08$ versus US $\$ 14,471.14 \pm 2,743.68$ for FESS. Sinusoplasty and FESS were similar in the improvement of postoperative symptoms; however, the sinusoplasty obtained better performance in patients' satisfaction and postoperative pain. ${ }^{4}$

Cazzavillan et al., ${ }^{7}$ consider it important also for patients to make an assessment of their own disease and their treatment, which is called "Patient Reported Outcome (RRP)", which refers to symptoms, perception of disease, Adherence to treatment and "Quality of Life Related to Disease (QVRD)." QVRD is the patient's own perception of the disease and its therapy in itself. The patient's point of view, because it is subjective, provides a description of his daily life quality. ${ }^{7}$ For rhinosinusitis, there are few questionnaires referring to QVRD in patients with this condition, the Rhinosinusitis Disability Index being the most used. This questionnaire contains 5 items, and shows that the disease significantly affects children's QVRD. The lack of questionnaires in children with rhinosinusitis may be due to this being, more often, an acute condition, while the usefulness of these is greater in CRS.

QVRD questionnaires were developed for children older than six years of age, however, one must take into account: I) the child's ability to read and understand the issues; II) the relevance of the item to the child; III) the child's ability to use the response options; IV) the ability to remember information in a given period of time; $\mathrm{V}$ ) the motivation of the child to complete the questionnaire. ${ }^{16}$ Thus, RRP begins to be considered mandatory in clinical evaluations, and it is recommended that it be included as an outcome, also in clinical trials. QVRD is the most widely used and well-known. ${ }^{7}$

There are many studies showing that each disease or comorbidity affects the QVRD of the pediatric population. In addition, by means of specific questionnaires, the effects of treatments can be more fully evaluated. ${ }^{17}$ RRP cannot replace the results, such as lung function in asthma and endoscopic evaluation in rhinosinusitis, but rather used in association with them. ${ }^{?}$

\section{Conclusion}

RSC is a common problem in the pediatric population and is often associated with other comorbidities such as asthma, allergic rhinitis, nasal polyposis, PDD, and cystic fibrosis. Initially, the treatment of CRS should be clinical medicated, through antibiotic therapy, symptomatic and treatment of the underlying cause. When therapeutic 
failure occurs and it persists, surgical treatment in children should be considered as an option, but should be according to the indications referring to each type of pathological condition, age group and be the least invasive possible.

Among the available surgical treatments, we conclude that adenoidectomy; FESS and BCS are effective, safe and with few complications, provided they are well indicated.

\section{Acknowledgements}

None.

\section{Conflict of interests}

The author states there is no conflict of interests.

\section{References}

1. Lai L, Hopp RJ, Lusk RP. Pediatric Chronic Sinusitis and Asthma: A Review. J Asthma. 43(10):719-725.

2. Ramadan Hassan H. Pediatric Sinusitis: Update. The Journal of Orolaryngology. 2005. p. 14-17.

3. LEO, Gualtiero; TRIULZI, Fabio; INCORVAIA, Cristoforo. Diagnosis of chronic rhinosinusitis. Pediatric Allergy and Immunology. Milan, p. 20-26. abr. 2012.

4. Carreirão Neto W, Pinna, Fábio De Rezende, Voegels Richard L. Sinusoplastia por Balão: Revisão da Literatura. Artigo de Revisão. 2008.

5. Vlastarakos PV, Fetta M, Segas JV, et al. Functional Endoscopic Sinus Surgery Improves Sinus-Related Symptoms and Quality of Life in Children With Chronic Rhinosinusitis: A Systematic Analysis and MetaAnalysis of Published Interventional Studies. Clin Pediatr (Phila). 2013;52(12):1091-1097.

6. Fiocchi A, Sarratud T, Bouygue GR, et al. Topical treatment of rhinosinusitis. Pediatr Allergy Immunol. 2007;18(Suppl 18):62-67.

7. Cazzavillan Alessandro. Management of chronic rhinosinusitis. Pediatric
Allergy and Immunology. 2012. p. 32-44.

8. Ramadan HH, Terrell AM. Balloon Catheter Sinuplasty and Adenoidectomy in Children with Chronic Rhinosinusitis. Ann Otol Rhinol Laryngol. 2010;119(9):578-582.

9. Sommer JU, Schafer K, Omran H, et al. ENT manifestations in patients with primary ciliary dyskinesia: prevalence and significance of otorhinolaryngologic co-morbidities. Eur Arch Otorhinolaryngol. 2011;268(3):383-388.

10. Cornet ME, Georgalas C, Reinartz SM, et al. Long-term results of functional endoscopic sinus surgery in children with chronic rhinosinusitis with nasal polyps. Rhinology. 2013;51(4):328-334.

11. El Sharkawy AA, Elmorsy SM, Eladl HM. Functional endoscopic sinus surgery in children: predictive factors of outcome. Eur Arch Otorhinolaryngol. 2012;269(1):107-111.

12. Aanaes K, von Buchwald C, Hjuler T, et al. The effect of sinus surgery with intensive follow-up on pathogenic sinus bacteria in patients with cystic fibrosis. Am J Rhinol Allergy. 27(1):e1-4.

13. Shirley SNP, Luc Louis Maurice M, Dirceu S. Rinossinusite na criança: Rhinosinusitis in children. Jornal de Pediatria. Rio de Janeiro. 1998. p. S31-S36.

14. Martinez FJ, Simon RH. Clinical implications of macrolide therapy in chronic sinopulmonary diseases. Curr Pharm Des. 2004;10(25):3095-110.

15. Anselmo-Lima WT, Sakano E, Tamashiro E, et al. Rhinosinusitis: evidence and experience. A summary. Braz $J$ Otorhinolaryngol. 2015;81(1):8-18.

16. Marco Antonio Cezario de Melo, Francis Z, Ulisses J. Cirurgia Endoscópica Nasossinusal: Endoscopic Sinus Surgery. Rev bras videocir. 2006;4(2):78-84.

17. Muntz HR, Lusk RP. Bacteriology of the Ethmoid Bullae in Children with Chronic Sinusitis. Arch Otolaryngol Head Neck Surg. 1991;117(2):179-181. 